\title{
Prof. Dr. habil. Ernst Pfeffer 65 Jahre
}

Am 31. Januar 2004 vollendet Univ. Prof. Dr. ERNST RICHARD PFEFFER sein 65. Lebensjahr. Aus diesem Anlass möchten wir unserem hochverehrten Kollegen sehr herzlich gratulieren.

Im Vorfeld dieses 65. Geburtstages fand ihm zu Ehren im Januar 2004 in Bonn ein wissenschaftliches Kolloquium zum Thema „Metabolismus von und Versorgung mit Stickstoff und Phosphor“ statt. Es würdigte das Lebenswerk von ERNST PFEFFER, dem Leiter des Institutes für Tierernährung der Rheinischen Friedrich - Wilhelms - Universität Bonn, dem er seit 1978 bis in die Gegenwart vorsteht.

Das Institut für Tierernährung bearbeitete unter seiner Leitung die Ernährung der verschiedenen Nutztierarten von der Forelle bis zum Rind, wobei ihre physiologischen Gegebenheiten ebenso wie ihr Bedarf und ihre Versorgungsempfehlung mit den verschiedenen Nährstoffen eine besondere Rolle spielten.

Im Mittelpunkt des Lebenswerkes von ERNST PFEFFER stand der Stickstoff- und Phosphorstoffwechsel und die Versorgung der landwirtschaftlichen Nutztiere mit diesen zwei Stoffgruppen. Es gelang ihm und seinen Mitarbeitern eine Fülle neuer Erkenntnisse zu erarbeiten, die in der landwirtschaftlichen Praxis Eingang fanden und deren Produktivität verbesserten. Das Ehrenkolloquium demonstrierte die zahlreichen Arbeiten des Jubilars auf diesen Spezialgebieten, den gegenwärtigen Wissensstand und ihre große nationale und internationale Anerkennung.

Fragmentarisch zeigte diese Veranstaltung Beispiele seines Wirkens als Forscher und zeugte davon, dass das Spektrum seiner Arbeiten sowohl die Grundlagenforschung als auch die praktische Tierernährung umfasst, wobei er bei der Wahl seiner Forschungsthemen stets die Praxiserfordernisse im Auge hatte. Das belegen auch sein umfangreiches publizistisches Wirken und eine intensive Öffentlichkeitsarbeit. Auf nationalen und internationalen Veranstaltungen war ERNST PFEFFER ein gern gesehener Referent und Gesprächspartner. Folgerichtig ist daher seine Mitarbeit in zahlreichen nationalen und internationalen Gremien und in mehreren Schriftleitungen wissenschaftlicher Zeitschriften gefragt. Auch in diesem, seinen Geburtstag würdigenden „Archiv für Tierzucht“, wirkt er in verdienstvoller Weise seit vielen Jahren im Redaktionskollegium.

Der Lebensweg des am 31. Januar 1939 in Leipzig geborenen Jubilars führte über die Volksschule in Hessen und das Humanistische Gymnasium in Hamburg-Groß Flotterbeck zum 
Abitur, dem sich eine landwirtschaftliche Lehre in Schleswig-Holstein anschloss, die er 1959 als Landwirtschaftsgehilfe abschloss. Von 1959 bis 1962 studierte ERNST PFEFFER in Weihenstephan und Göttingen Agrarwissenschaften. Er beendete seine Studienzeit als Diplomlandwirt und erhielt ein Promotionsstipendium an der Universität Göttingen. 1964 promovierte er zum Dr. sc. agr. mit dem Thema „Langfristige Untersuchungen an Milchkühen über den Einfluss einer natriumarmen Ernährung auf das Plasmavolumen und auf den Chloridstoffwechsel“. Im Anschluss daran arbeitete er als Stipendiat der Deutschen Forschungsgemeinschaft im Department of Agricultural Biochemistry der University of Newcastle upon Tyne in Großbritanien und habilitierte sich 1968 mit dem Thema „Untersuchungen über Mineralstoffbewegungen im Verdauungskanal von ausgewachsenen Hammeln“, (venia legendi: Tierphysiologie und Tierernährung). Der Habilitation schlossen sich eine Universitätsdozentur und -professur für Leistungsphysiologie in Göttingen an, wo er von 1970 bis 1978 wirkte. Im Jahr 1978 wurde er als ordentlicher Professor und Leiter des Instituts für Tierernährung der Universität Bonn als Nachfolger von RICHARD MÜLLER berufen. Als Hochschullehrer und Forscher wirkte er an diesem Institut über ein Vierteljahrhundert sehr erfolgreich. Seine fachliche Kompetenz und hohes Engagement verhalfen diesem noch relativ jungen Institut zu hohem nationalen und internationalem Ansehen.

Seine besondere Fürsorge gilt seinen Studenten und so konnte er während seiner langen Wirkungszeit als Hochschullehrer viele junge Studenten für sein Fach begeistern und zur Mitarbeit gewinnen. Bei ihnen war er sehr beliebt, da sie in ihm immer einen anspruchsvollen, hilfreichen und freundlichen Ratgeber fanden. Er lebt ihnen in bester Weise den modernen Hochschullehrer und Forscher vor. Groß ist die Zahl seiner Diplomanden, Doktoranden und Habilitanden, die sich den heutigen Glückwünschen anschließen.

Fachkollegen des In- und Auslandes danken dem Jubilar für eine sehr gute umfangreiche interdisziplinäre Arbeit sowie die verlässliche Zusammenarbeit und glauben, dass sein Kalender immer noch voll ist mit Terminen und Aufgaben.

Wir, viele Kollegen, Schüler, Mitarbeiter und der Laudator wünschen ERNST PFEFFER noch viele schöne, erfolgreiche, glückliche und gesunde Jahre im Kreise seiner Familie.

Manfred Anke, Jena 